\title{
Status assessment of the Endangered snow leopard Panthera uncia and other large mammals in the Kyrgyz Alay, using community knowledge corrected for imperfect detection
}

\author{
Julia Taubmann, Koustubh Sharma, Kubanychbek Zhumabai Uulu \\ James E. Hines and Charudutt Mishra
}

\begin{abstract}
The Endangered snow leopard Panthera uncia occurs in the Central Asian Mountains, which cover c. 2 million $\mathrm{km}^{2}$. Little is known about its status in the Kyrgyz Alay Mountains, a relatively narrow stretch of habitat connecting the southern and northern global ranges of the species. In 2010 we gathered information on current and past (1990, the last year of the Soviet Union) distributions of snow leopards and five sympatric large mammals across $14,000 \mathrm{~km}^{2}$ of the Kyrgyz Alay. We interviewed 95 key informants from local communities. Across $49400-\mathrm{km}^{2}$ grid cells we obtained 1,606 and 962 records of species occurrence (site use) in 1990 and 2010, respectively. The data were analysed using the multi-season site occupancy framework to incorporate uncertainty in detection across interviewees and time periods. High probability of use by snow leopards in the past was recorded in $>70 \%$ of the Kyrgyz Alay. Between the two sampling periods $39 \%$ of sites showed a high probability of local extinction of snow leopard. We also recorded high probability of local extinction of brown bear Ursus arctos ( $84 \%$ of sites) and Marco Polo sheep Ovis ammon polii ( $47 \%$ of sites), mainly in regions used intensively by people. Data indicated a high probability of local colonization by lynx Lynx lynx in $41 \%$ of the sites. Although wildlife has declined in areas of central and eastern Alay, regions in the north-west, and the northern and southern fringes appear to retain high conservation value.
\end{abstract}

Keywords Capra sibirica, local knowledge, Lynx lynx, occupancy modelling, Ovis ammon polii, Panthera uncia, Ursus arctos

Julia Taubmann* Wildlife Ecology and Management, University of Freiburg, Tennenbacher Straße 4, 79106 Freiburg, Germany

Koustubh Sharma $†$ (Corresponding author), Kubanychbek Zhumabai Uulu‡ and Charudutt Mishra $†$ Snow Leopard Trust, 4649, Sunnyside Avenue, \#325 North Suite, Seattle, USA

E-mail koustubhsharma@gmail.com, koustubh@snowleopard.org

James E. Hines United States Geological Survey, Patuxent Wildlife Research Center, Laurel, Maryland 20708, USA

*Previously at: Department of Ecology, University of Mainz, Germany, and Snow Leopard Trust, Seattle, USA

$\dagger$ Also at: Nature Conservation Foundation, 3076/5, IV Cross, Gokulam Park, Mysore, India

¥Also at: Snow Leopard Foundation, Bishkek, Kyrgyzstan

Received 30 September 2014. Revision requested 7 November 2014.

Accepted 2 April 2015. First published online 9 September 2015.
To view supplementary material for this article, please visit http://dx.doi.org/10.1017/So030605315000502

\section{Introduction}

The global decline and increasing risk of extinction of large mammals, especially large carnivores, is driven by a combination of their intrinsic life-history traits, environmental factors and anthropogenic pressures (Brashares, 2003; Cardillo et al., 2004, 2005; Karanth et al., 2011; Pillay et al., 2011). Ultimately, the persistence, decline or changes in distribution of a species may be perceived as the outcome of local colonizations and extinctions, and understanding colonization-extinction dynamics can be valuable for conservation planning. Assessment and monitoring of the conservation status of species can be challenging, however, particularly for elusive and rare carnivores such as the snow leopard Panthera uncia (Jackson \& Hunter, 1996; McCarthy et al., 2008). Long-term data on abundance patterns are desirable but methods such as transect surveys, camera-traps and non-invasive genetic sampling are cost intensive and time consuming, making them less suitable for monitoring wildlife status over large areas (van der Hoeven et al., 2004; Jackson et al., 2006; McCarthy et al., 2008; Janečka et al., 2011; Zeller et al., 2011; Sharma et al., 2014).

Occupancy is a state variable that describes the probability of the sampled sites being occupied by a species (MacKenzie et al., 2003). It has been used to estimate species occurrence, distribution and habitat selection for a variety of taxa (Bailey et al., 2004, 2007; Cabeza et al., 2004; MacKenzie \& Royle, 2005; Smith et al., 2007; Ruell et al., 2009). Site occupancy estimates account for false absence data (i.e. when a species is present in an area but is not detected during sampling), a problem encountered commonly in relation to rare species (MacKenzie et al., 2005). With spatio-temporal data, the multi-season occupancy framework provides a useful means of estimating local colonization and extinction probabilities across two or more sampling periods (MacKenzie et al., 2003).

Local people living in areas adjoining or overlapping with wildlife habitats represent a source of potentially valuable information on the occurrence of species and the status of 
biodiversity (Lunney et al., 2009; Pillay et al., 2011; Zeller et al., 2011). The knowledge of local residents about prominent wild species, recorded in interviews, can help assess species distributions and status over large landscapes, with moderate effort (Lunney et al., 2009). However, the detection of an elusive species is seldom perfect, with a reasonable possibility that it may be present in a sampling unit but remain undetected, even over extended periods of time. Data from multiple interviewees reporting the detection or nondetection of a species in a sampling unit, when analysed using a site occupancy framework, can help address the problem of non-detection. People can provide information about past occurrence (site use) of a target species, and such data can inform our understanding of large-scale spatiotemporal trends and facilitate estimation of the probabilities of extinction and colonization under specific model formulations (Karanth et al., 2011; Pillay et al., 2011; Zeller et al., 2011).

The Endangered snow leopard occurs in the Central Asian Mountains, which span c. 2 million $\mathrm{km}^{2}$ over 12 countries. The northern and southern parts of the snow leopard's global range are connected by the Kyrgyz Alay Mountains (Fig. 1). In the 1980s the Kyrgyz Republic and neighbouring Tajikistan were estimated to have $1,200-1,400$ snow leopards, representing $>20 \%$ of the global population. This population is suspected to have declined by $75 \%$ in the 1990s after the fall of the Soviet Union (Koshkarev \& Vyrypaev, 2000). Human and livestock populations increased, intensifying grazing pressure, poaching and the demand for fuel (Murray, 2008). Thus changes in the distribution and populations of wildlife may have occurred following the political and economic changes that accompanied the breakdown of the Soviet Union.

The Alay Mountains, located in southern Kyrgyzstan along the border with Tajikistan, represent the northern rim of the Pamir-Alay Mountain system. As the link between the Himalaya-Hindukush in the south and the Tien Shan and Altai mountain chains in the north, they have high biogeographical and conservation importance. Although snow leopards have been studied in other parts of Kyrgyzstan (e.g. McCarthy et al., 2008; Jumabay-Uulu et al., 2014), little research has been done on the wildlife of Alay. Snow leopards were not mentioned in early literature such as the monograph Mammals of Kyrgyzia (Yanushevich et al., 1972), or in more recent publications by Vorobeev \& Van Der Ven (2003) and Farrington (2005), which reported the presence of other species (e.g. ibex Capra sibirica and Marco Polo sheep Ovis ammon polii) in the Alay. Biodiversity surveys based on interviews with local residents provided no information on snow leopards but reported their presence in the Tajik Pamir Mountains (Sagimbaev, 2007; Murray, 2008). Izumiyama et al. (2009) suggested that snow leopards may have gone extinct locally, but their methods did not take into consideration the possibility that the species may have been present but undetected. Watanabe et al. (2010) reported that $21-27 \%$ of interview respondents among local people in Alay knew of the existence of snow leopards and argali in the region, and that there was significant anthropogenic disturbance in the Kyrgyz Alay, in the form of livestock grazing, and legal and illegal hunting, in contrast to low densities of people and livestock on the Tajik side. A sizeable ibex population was reported in the upper Alay Mountains (Murray, 2008), indicating that wild ungulates and carnivores may still survive in remote areas.

We used interview-based site-occupancy modelling to assess the past (1990; the last year of the Soviet Union) and current (2010) status of the snow leopard and sympatric wildlife, including lynx Lynx lynx, brown bear Ursus arctos, Marco Polo sheep, wolf Canis lupus and ibex, in the Kyrgyz Alay. Our objective was to conduct a systematic assessment of the changes in distribution and conservation status of the snow leopard, its prey, and sympatric large carnivores in the region. We also tested the possibility of co-occurrence, or avoidance in the case of lynx and snow leopard, in the study area. Our data were recorded during interviews of key informants in local communities, and corrected for imperfect detection using maximum-likelihood-based models. We provide a spatially explicit assessment of local extinction and colonization of snow leopards and other sympatric species in this landscape, and identify hotspots for focusing future survey and conservation efforts.

\section{Study area}

Our study was conducted across the Alay Mountains, the Alay Valley, and the northern slopes of the Trans-Alay range, in an area of c. 14,000 $\mathrm{km}^{2}$ bounded by the Kyrgyz-Tajik border in the south and high mountains in the north (Fig. 1). The Alay Valley divides the Alay Mountains and the Pamir Mountains, and is the largest inter-mountain depression in the Pamir-Alay Mountain System. Most of the settlements in the study area are located in valleys. The region to the north of the study area borders the densely populated Fergana Valley. Human population density in the Alay region was reported to be c. 5 per $\mathrm{km}^{2}$ (Asykulov, 2008) and is expected to have increased by c. $25 \%$ during 1989-2009 (National Statistical Committee, 2010). Livestock rearing is the predominant livelihood activity, with additional resources provided by hunting (Asykulov, 2008). The region has a continental climate, with a mean temperature of $-17^{\circ} \mathrm{C}$ in winter and $15^{\circ} \mathrm{C}$ in summer, and an annual mean of $6.5^{\circ} \mathrm{C}$ (Majit, 2012). Annual precipitation is c. $275 \mathrm{~mm}$, with a mean of $40 \mathrm{~mm}$ per month in summer and $90 \mathrm{~mm}$ per month in winter; in the valleys mean precipitation is $24 \mathrm{~mm}$ per month in summer and $36 \mathrm{~mm}$ per month in winter. Snowfall occurs 

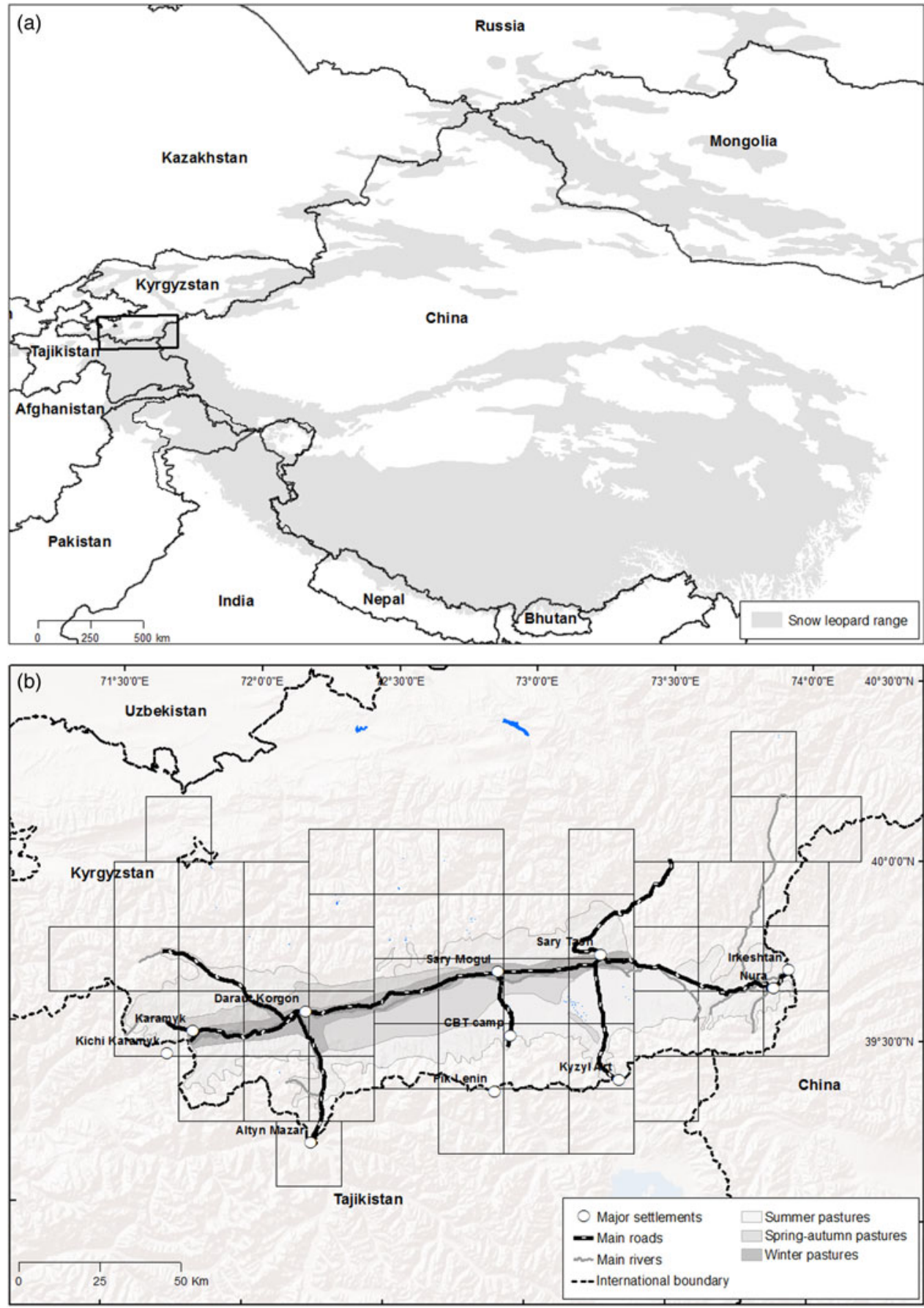

FIG. 1 (a) Global distribution of the snow leopard Panthera uncia, and the location of the study area in the Alay region of the Kyrgyz Republic. (b) The sampling grid used in site-occupancy surveys for snow leopard, ibex Capra sibirica, Marco Polo sheep Ovis ammon polii, wolf Canis lupus, brown bear Ursus arctos and lynx Lynx lynx. mostly during November-March, with rain during May and June. The snow line follows a gradient of 3,500 to $4,400 \mathrm{~m}$ from west to east. Vegetation may be categorized as semidesert, steppe, meadow steppe, subalpine and alpine meadow steppe, and spruce Picea forests. Oil and mineral extraction, gold mining and road construction are imminent threats to wildlife. A Pamir-Alay Trans-boundary Conservancy Area was proposed under a grant from the European Union (Murray, 2008) but has yet to be implemented (Snow Leopard Working Secretariat, 2013).

\section{Methods}

\section{Sampling approach and data collection}

The study area was divided into $49400-\mathrm{km}^{2}$ square sampling units, each approximating the mean home range of the snow leopard (c. $350 \mathrm{~km}^{2}$; McCarthy et al., 2010).

JT conducted interview-based site-occupancy surveys during October-November 2010, assisted by an interpreter. The surveys included questions about sightings and evidence 
of snow leopard, ibex, Marco Polo sheep, wolf, brown bear and lynx, and the extent of wildlife hunting and livestock depredation. We considered instances of hunting, direct sightings, and clearly identifiable signs (pugmarks, scrapes) to be species detections. We also included questions about the interviewee's experience, profession and time spent in the area of interest annually, to model as potential factors influencing the probability of the interviewee detecting the presence of wildlife and retaining the information.

Detailed conversations with interviewees and verification of their knowledge are necessary to obtain reliable information in a structured interview (White et al., 2005; Zeller et al., 2011). We started the interviews by introducing ourselves and declaring that all information provided by the interviewee would be kept anonymous. The reliability of the interviewee's knowledge was tested by their description and identification of pictures of snow leopards and sympatric wildlife. Most of the interviews were conducted in the interviewee's house, in the presence of their family. In this familiar atmosphere, which was comfortable for both interviewees and interviewers, there were often extensive discussions about snow leopards, wildlife and people's needs and concerns, yielding highquality information.

The efficiency of the fieldwork was maximized by conducting interviews in autumn and winter, when most of the interviewees were available at home. We conducted a total of 95 independent interviews in 24 of 28 villages in the study area. Heavy snowfall prevented access to four villages but sufficient information about species in the area was obtained from people in neighbouring villages. Statistically, each interviewee represented a replicate for one or more sampling units with which he or she was familiar (i.e. the area of knowledge; Zeller et al., 2011). The interviewees defined their own area of knowledge, from which we assigned the information to specific sampling units. Replicate coverage of the sampling units comprised 3-18 respondents for 2010 and 3-26 respondents for 1990. If an interviewee had been inactive during one of the time periods for which we sought information, that time period was excluded from the data set. Interviewees included 92 men and three women aged 19-89 years. The interviewees reported spending 12-330 days per year in their area of knowledge in 2010 and 4-365 days per year in 1990 .

We relaxed the assumption of closure of sites to changes in occupancy during the sampling periods, and the occupancy results were treated as probabilities of site use (MacKenzie \& Nichols, 2004) in $1990(\psi 1)$ and $2010(\psi 2)$. We estimated the probabilities of local extinction ( $\varepsilon$; a site used in 1990 is no longer used) and local colonization ( $\gamma$; a site that was not used in 1990 is used currently) for all sampled units. In the occupancy models we used $\varepsilon, \gamma$ and the probability of past site use $(\psi 1)$ recursively to estimate the current probability of site use $\left(\psi_{2}\right)$ in all sampled units. People's memories vary and their ability to report precisely about events that occurred during a particular timeframe may decrease over time. To address this we also modelled for variation in detection probabilities between the two time periods.

We created five spatial layers for the covariates affecting site use and extinction probabilities (Supplementary Material 1), using the Spatial Analyst tool in ArcGIS v. 10.0 (ESRI, Redlands, USA). These layers included vegetation cover (normalized difference vegetation index) and four topographic covariates (altitude, slope, ruggedness and aspect), and were created using remotely sensed satellite imagery (Global Land Cover Facility, 2010) and Shuttle Radar Topography Mission data (CGIAR-CSI, 2004), respectively. The vector ruggedness measure of the study area was created using a geoprocessing script developed by Sappington et al. (2007). Covariate pixel values were averaged for each sampling unit. As a measure of human disturbance we used the distance of each sampling unit from the nearest settlement as site covariate.

\section{Data analysis}

For 1990 and 2010 we recorded 1,606 and 962 reports of detection or non-detection of species across the 49 grid cells, respectively. Multiple reports from within a sampling grid cell by each observer were merged and assigned a value of 1 or 0 , representing detection or non-detection. This resulted in 607 and 395 information points for the matrix representing detection histories of various species for 1990 and 2010, respectively.

Site covariates that could influence probabilities of site use, extinction and colonization were incorporated in the occupancy models by means of logistic insertion. Survey covariates that could influence the probability of detection (interviewee profession, experience, days spent in their area of knowledge) were also incorporated. Profession was categorized as local hunter (profi), herder or herder \& local hunter (prof2), or other (prof 3 ). Individuals who reportedly hunted periodically were categorized as hunters. We tested the effect of time period in our models to address possible variation between the detection probabilities for 1990 and 2010.

The probabilities of species site use, detection, and local extinction and colonization were modelled using PRESENCE v. 6.4 (Hines, 2006). Changes in site use, detection, extinction and colonization rates between the sampling years were modelled using a multi-season approach (MacKenzie et al., 2003). We used Akaike weights to rank our set of models (Anderson \& Burnham, 2002; Symonds \& Moussalli, 2011), and we model-averaged estimates of site use, colonization, extinction and detection probabilities using those models that cumulatively accounted for $95 \%$ of the total model weight.

By plotting the model-averaged estimates of $\psi 1, \varepsilon, \gamma$ and $\psi 2$ on the sampling units in ArcGIS v.10.2.2 we were able to 
create probabilistic maps for species distributions in 1990 and 2010, as well as colonization and extinction patterns. To identify sites for more detailed surveys and targeted conservation efforts, we summed the pixel values of current probability of site use for all species and divided the sampling units into three categories of conservation value. The sampling units with top, middle and lowest one-third pixel values were categorized as having high, medium and low conservation value, respectively.

Our analysis suggested contrasting trends between snow leopard and lynx, and therefore we also ran co-occurrence models (MacKenzie et al., 2004) on these species to estimate the probability of occurrence of one given the probability of presence or absence of the other. We ran these models on the 2010 data for the two species to determine if there was any indication of mesopredator release. For the scenario where lynx benefitted from the absence of snow leopard we expected $\psi^{\mathrm{Ba}}$ (the probability of an area being used by species $\mathrm{B}$, given the absence of species $\mathrm{A}$ ) to be high. However, we expected the value of $\psi^{\mathrm{BA}}$ (the probability of an area being used by species $\mathrm{B}$, given presence of species A) to be high if the two species co-occurred.

\section{Results}

Interviewees reported snow leopard presence in 26 sampling units in total over both time periods. Detections were reported in 24 sampling units in 1990 and 11 in 2010. Lynx, brown bear and Marco Polo sheep were detected in 24, 24 and 22 sampling units, respectively, in 1990 and in 35, 12 and 9 sampling units in 2010. Naïve occupancy estimates (assuming all sites being used by the species in a specific period were reported) were 0.49 in 1990 and 0.22 in 2010 for snow leopard, 0.29 and 0.10 for brown bear, 0.29 in both periods for lynx, and 0.33 and 0.37 for Marco Polo sheep. Wolf and ibex were detected in all sampling units in both sampling periods, precluding any need for estimating site-use probability; hence these two species were excluded from further analyses.

For each model, the model weight indicates the probability of it being the best model in the set (Table 1). The number of models whose Akaike weights summed to $\geq 0.95$ varied with species ( 13 for Marco Polo sheep, 20 for snow leopard).

We found that the detection probability varied between the two time periods, whereas profession, experience and number of days spent in the area of knowledge had a relatively smaller but evident effect (Table 2). Profession and days spent in the area of knowledge affected the detection probability of Marco Polo sheep, followed by the time period. Time period, followed by days spent in the area of knowledge and work experience improved the probability of detection of brown bear. For lynx, detectability was most influenced by experience and days spent in the field, followed by time period and profession.

\section{Site use and local extinction/colonization probabilities}

We were able to test the effects of six site covariates on estimated probabilities of sites used, and of their local colonization and extinction, for the four species (Table 2). The probability of site use by snow leopards suggested a moderate to high presence in the western, northern and eastern regions of Alay (Fig. 2a) and ranged between $0.07 \pm$ SE 0.09 and $0.96 \pm$ SE 0.04. Apart from some regions of central Alay that were used as seasonal pastures by herders, there was a high probability of site use by snow leopards in 1990 ( $>0.5$ in $73 \%$ of sampled units), with high distribution probability primarily characterized by relatively lower mean altitudes $\left(\beta_{\text {altitude }}=-1.82 \pm\right.$ SE o.86) and steep slopes $\left(\beta_{\text {slope }}=2.15 \pm\right.$ SE 0.86). There was also some evidence of the effect of ruggedness and a possible interaction between ruggedness and slope in some of the lower-ranking models (Table 1). Distance from settlements and vegetation index had no discernible effect on the probability of sites being used by snow leopards. In $39 \%$ of the sampled sites there was a high probability $(>0.5)$ that the snow leopard had gone extinct locally. Most local extinctions may have occurred towards the central and eastern parts of Alay, around sites with relatively gentle slopes, which tend to be areas of high human use in the form of settlements, grazing pastures and infrastructural development. In contrast $61 \%$ of the sampling units, mostly towards the north and south-west regions of Alay, had a low probability of extinction $(<0.5)$, indicating that snow leopard populations persist in these regions. None of the sampled units across the study area showed any evidence of local colonization. The probability of site use in 2010 was between $0.00 \pm$ SE 0 and 0.96 \pm SE 0.04 , and $53 \%$ sites had a high probability $(>0.5)$ of site use. This was higher towards the northern, western and south-western edges of Alay, in areas that were steeper and relatively less accessible from settlements and pastures (Fig. 2b).

In 1990 lynx were distributed throughout the Alay region, with lower probabilities of site use around the centre (Fig. 2c). None of the sampled units showed a high probability $(>0.5)$ of past site use. Data for 2010 indicated a high probability of site use in $41 \%$ of the sampling units (Fig. $2 \mathrm{~d}$ ). Although a marginal net decline was indicated, lynx was the only species that appeared to have colonized new areas. The probability of local extinction was between $0.04 \pm$ SE 0.24 and $0.47 \pm$ SE 0.85 across the region, whereas the probability of local colonization was between $0.00 \pm$ SE 0.69 and $0.85 \pm$ SE 0.22. The probability of local colonization was slightly higher near settlements and towards the north-east and north-west of the region. There appeared to be a spatial pattern in the probabilities of local colonization by lynx and local extinction of snow leopard, with a correlation coefficient of 0.28 . However, our estimates regarding lynx were relatively imprecise because of high variance. 
TABLE 1 Top multi-season models for snow leopard Panthera uncia, bear Ursus arctos, lynx Lynx lynx and Marco Polo sheep Ovis ammon polii, ranked in ascending order of AIC.

\begin{tabular}{|c|c|c|c|c|c|}
\hline Model $^{*}$ & AIC & $\Delta \mathrm{AIC}$ & Akaike weight & No. of parameters & $-2 \log L$ \\
\hline \multicolumn{6}{|l|}{ Snow leopard } \\
\hline psi(Alt + Slp), gamma(.), eps(Slp), p(Seasonal) & 516.58 & 0 & 0.0955 & 8 & 500.58 \\
\hline psi(Alt + Slp), gamma(.), eps(Slp), p(Prof Seasonal) & 516.63 & 0.05 & 0.0931 & 10 & 496.63 \\
\hline psi(Alt + Slp $)$, gamma(.), eps(Slp), p(Seasonal + DaysAoK) & 516.82 & 0.24 & 0.0847 & 9 & 498.82 \\
\hline psi(Alt + Slp), gamma(.), eps(Slp), p(DaysAoK) & 517.07 & 0.49 & 0.0747 & 8 & 501.07 \\
\hline psi(Alt + Slp $), \operatorname{gamma}()$, eps(Slp), p(Experience) & 517.07 & 0.49 & 0.0747 & 8 & 501.07 \\
\hline psi(Alt + Slp $), \operatorname{gamma}()$, eps(Slp), p(Seasonal + Experience $)$ & 517.12 & 0.54 & 0.0729 & 9 & 499.12 \\
\hline psi(Alt + Slp), gamma(.), eps(Slp), p() & 517.18 & 0.6 & 0.0707 & 7 & 503.18 \\
\hline psi(Alt + Slp), gamma(.), eps(Slp), p(Prof) & 517.34 & 0.76 & 0.0653 & 9 & 499.34 \\
\hline psi(Alt + RgdXSlp), gamma(.), eps(Slp), p(Seasonal) & 518.24 & 1.66 & 0.0416 & 10 & 498.24 \\
\hline psi(Alt + Slp), gamma(.), eps(Rgd), p(Seasonal) & 518.37 & 1.79 & 0.039 & 8 & 502.37 \\
\hline \multicolumn{6}{|l|}{ Bear } \\
\hline psi, gamma(), eps(Slp), p(AoK Seasonal)’ & 288.79 & 0 & 0.1394 & 7 & 274.79 \\
\hline psi, gamma(), eps(Slp), p(AoK + Exp Seasonal)' & 289.06 & 0.27 & 0.1218 & 8 & 273.06 \\
\hline psiz(Slp), gamma(), eps(Slp), p(AoK Seasonal)' & 289.65 & 0.86 & 0.0907 & 8 & 273.65 \\
\hline psi(Rgd), gamma(), eps(Slp), p(AoK Seasonal)' & 289.69 & 0.9 & 0.0889 & 8 & 273.69 \\
\hline psi, gamma(), eps(), p(AoK Seasonal) & 289.73 & 0.94 & 0.0871 & 6 & 277.73 \\
\hline psi, gamma(), eps(Rgd), p(AoK Seasonal)' & 290.15 & 1.36 & 0.0706 & 7 & 276.15 \\
\hline psi, gamma(), eps(Slp), p(Seasonal)' & 290.38 & 1.59 & 0.0629 & 6 & 278.38 \\
\hline psi, gamma(), eps(Slp + Rgd), p(AoK Seasonal)' & 290.44 & 1.65 & 0.0611 & 8 & 274.44 \\
\hline psi(Alt), gamma(), eps(Slp), p(AoK Seasonal)' & 290.59 & 1.8 & 0.0567 & 8 & 274.59 \\
\hline psi, gamma(), eps(Slp), p(Exp Seasonal) & 290.82 & 2.03 & 0.0505 & 7 & 276.82 \\
\hline \multicolumn{6}{|l|}{ Lynx } \\
\hline psi(Alt), gamma(Alt), eps(.), p(Exp + AoK)' & 372.45 & 0 & 0.1588 & 8 & 356.45 \\
\hline psi(Alt + Rgd), gamma(Alt), eps(.), p(Exp + AoK)' & 372.94 & 0.49 & 0.1243 & 9 & 354.94 \\
\hline psi(Alt), gamma(Alt), eps(Slp), p(Exp + AoK)' & 373.17 & 0.72 & 0.1108 & 9 & 355.17 \\
\hline psi(Alt + Rgd $), \operatorname{gamma}($ Alt $), \operatorname{eps}($ Slp'), p(Exp + AoK)' & 373.48 & 1.03 & 0.0949 & 10 & 353.48 \\
\hline psi(Alt), gamma(Alt), eps(Alt), p(Exp + AoK)' & 373.56 & 1.11 & 0.0912 & 9 & 355.56 \\
\hline psi(Alt), gamma(Alt), eps(.), p(Exp Seasonal)' & 373.69 & 1.24 & 0.0854 & 8 & 357.69 \\
\hline psi(Alt + Rgd $),$ gamma(Alt), eps(Alt), p(Exp + AoK)' & 374.21 & 1.76 & 0.0659 & 10 & 354.21 \\
\hline psi(Alt + Rgd), gamma(Alt), eps(Slp'), p(Exp)' & 374.65 & 2.2 & 0.0529 & 9 & 356.65 \\
\hline psi(Alt + Rgd), gamma(Alt), eps(Slp'), p(AoK)' & 375.41 & 2.96 & 0.0362 & 9 & 357.41 \\
\hline psi(Alt), gamma(Alt), eps(.), p(Exp + AoK + Prof)' & 375.97 & 3.52 & 0.0273 & 10 & 355.97 \\
\hline \multicolumn{6}{|l|}{ Marco Polo sheep } \\
\hline psi(SlpXRgd), gamma(), eps(Dist_S), p(Prof + AoK) & 251.9 & 0 & 0.1884 & 11 & 229.9 \\
\hline psi(Alt + Rgd), gamma(), eps(Dist_S $),$ p(Prof + AoK $)$ & 252.26 & 0.36 & 0.1574 & 10 & 232.26 \\
\hline psi(Alt + Rgd), gamma(), eps(Dist_S), p(Prof) & 252.81 & 0.91 & 0.1195 & 9 & 234.81 \\
\hline psi(Rgd), gamma(), eps(Dist_S), p(Prof + AoK) & 253.18 & 1.28 & 0.0994 & 9 & 235.18 \\
\hline psi(Alt + Rgd), gamma(), eps(Rgd), p(Prof + AoK $)$ & 253.4 & 1.5 & 0.089 & 10 & 233.4 \\
\hline psi(Alt + Slp), gamma (), eps(Dist_S $), p($ Prof $)$ & 254 & 2.1 & 0.0659 & 9 & 236 \\
\hline psi(Alt + Rgd $),$ gamma(), eps(Dist_S $),$ p(Prof + AoK Seasonal $)$ & 254.25 & 2.35 & 0.0582 & 11 & 232.25 \\
\hline psi(Alt + Rgd $),$ gamma( $),$ eps(Dist_S $),$ p(Prof Seasonal $)$ & 254.81 & 2.91 & 0.044 & 10 & 234.81 \\
\hline psi(Slp + Rgd), gamma(), eps(Dist_S), p(Prof + AoK) & 255.17 & 3.27 & 0.0367 & 10 & 235.17 \\
\hline psi(Alt), gamma(), eps(Dist_S), p(Prof + AoK) & 255.53 & 3.63 & 0.0307 & 9 & 237.53 \\
\hline
\end{tabular}

${ }^{\star}$ Site covariates: Alt, altitude; Rgd ${ }^{\star}$ Slp, ruggedness ${ }^{\star}$ slope (vector ruggedness measure); Slp, slope; Rgd, ruggedness; NDVI, normalized difference vegetation index; Asp, aspect north; Dist, distance to settlements. Survey covariates: Seasonal, seasonal effects; Prof1, professional hunter; Prof2, professional herder and herder-hunter; Prof 3 , professional other; Exp, working experience of interviewee; Days, days per year spent in the interviewee's area of knowledge.

Past distribution of brown bears was uniform across Alay (Fig. 2e), and the probability of site use by brown bears in 1990 in the sampled units was between $0.36 \pm$ SE 0.17 and $0.48 \pm$ SE 0.16 . Up to $84 \%$ of sites showed a high probability $(>0.5)$ of local extinction. The current distribution appears to be restricted to the north and north-west regions of Alay, including patches near the southern border with Tajikistan
(Fig. 2f). There appears to be a higher probability of local extinction in areas with lower slope and ruggedness values, and close to settlements (Table 2).

For Marco Polo sheep the probability of past site use was high $(>0.5)$ for $47 \%$ of sites, with population strongholds centred along the border of Kyrgyzstan and Tajikistan, and parts of central Alay (Fig. 2g). The sheep were more likely 
TABLE 2 Estimates of $\beta$-coefficients of covariates $\psi$ (occupancy), $\gamma$ (colonization), $\varepsilon$ (extinction) and P (detection probability) for the top-scoring multi-season models of snow leopard, bear, lynx and Marco Polo sheep, and the relative variable importance for all covariates. Blank cells indicate that those covariates were not used for the top model.

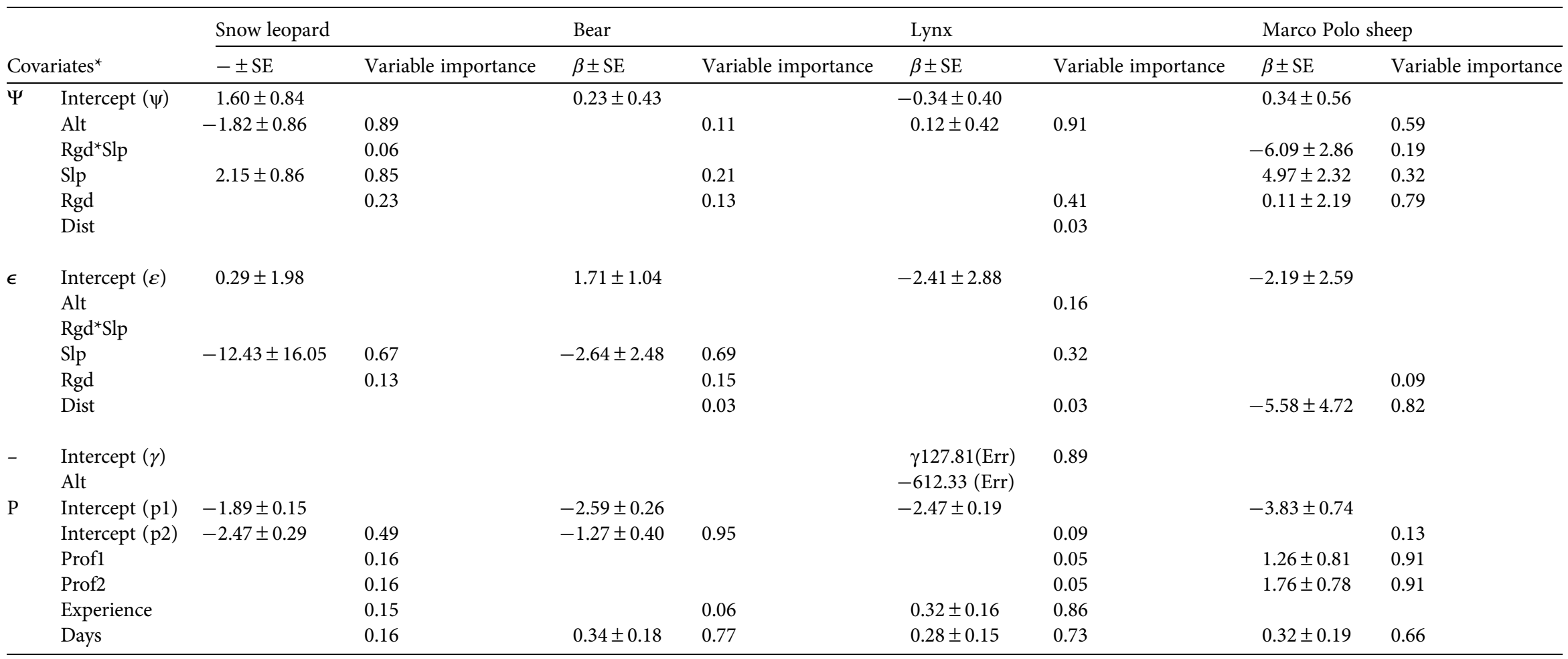

${ }^{\star}$ Site covariates (for $\psi$ and $\varepsilon$ ): Alt, altitude; Rgd ${ }^{\star}$ Slp, interaction between ruggedness \& slope; Slp, slope; Rgd, ruggedness; Dist, distance from the nearest settlement. Survey covariates (for P): Intercept (p1) and Intercept (p2), representing time periods 1990 and 2010, respectively; Prof1, part-time hunter; Prof2, full-time hunter; Experience, working experience of interviewee; Days, days per year spent by the interviewee in the area of knowledge. 

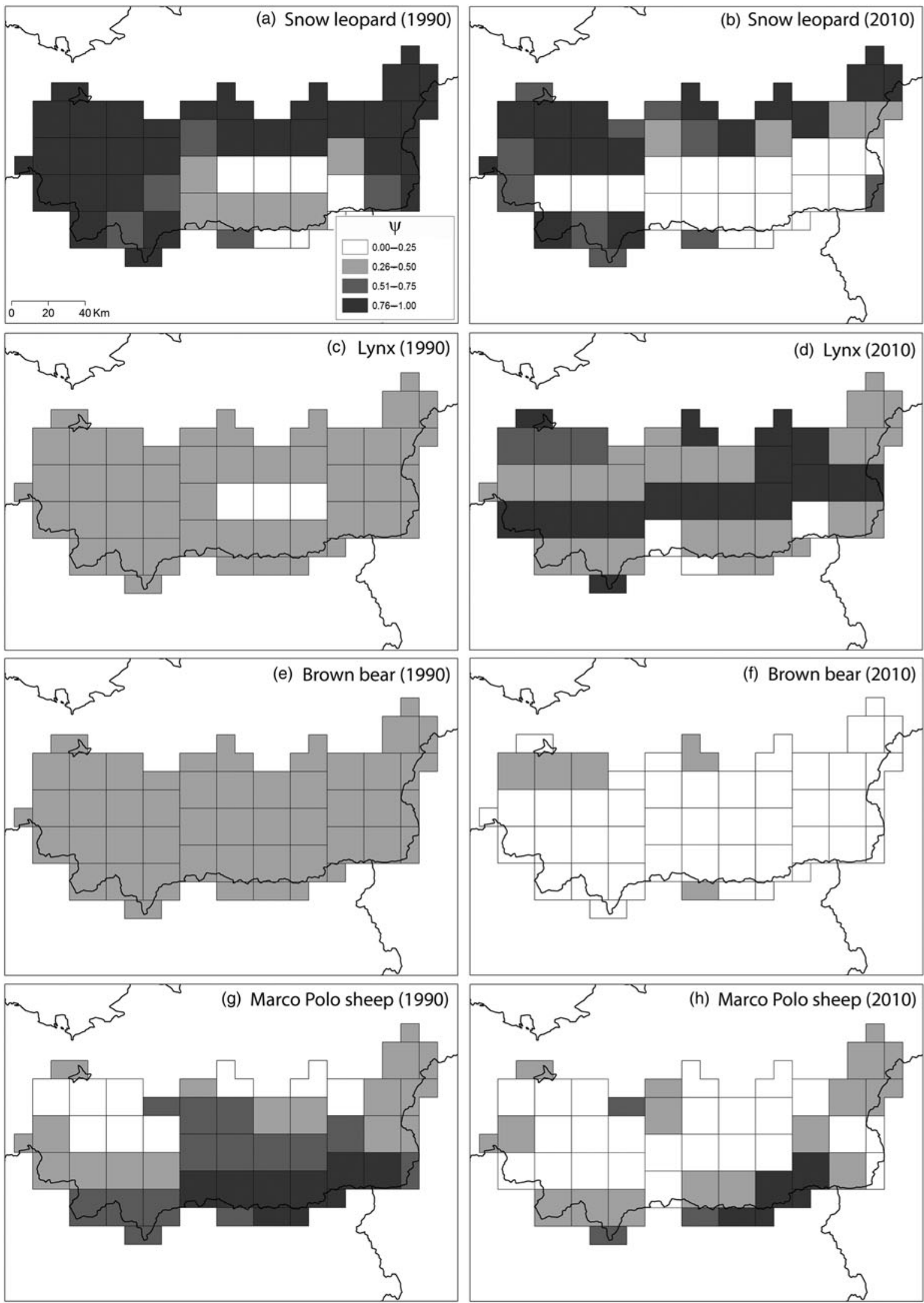

Fig. 2 Estimated probabilities of site use in 1990 and 2010 for snow leopard (a \& b), lynx (c \& d), brown bear (e \& f) and Marco Polo sheep ( $\mathrm{g} \& \mathrm{~h}$ ) in the Kyrgyz Alay Mountains (Fig. 1). 


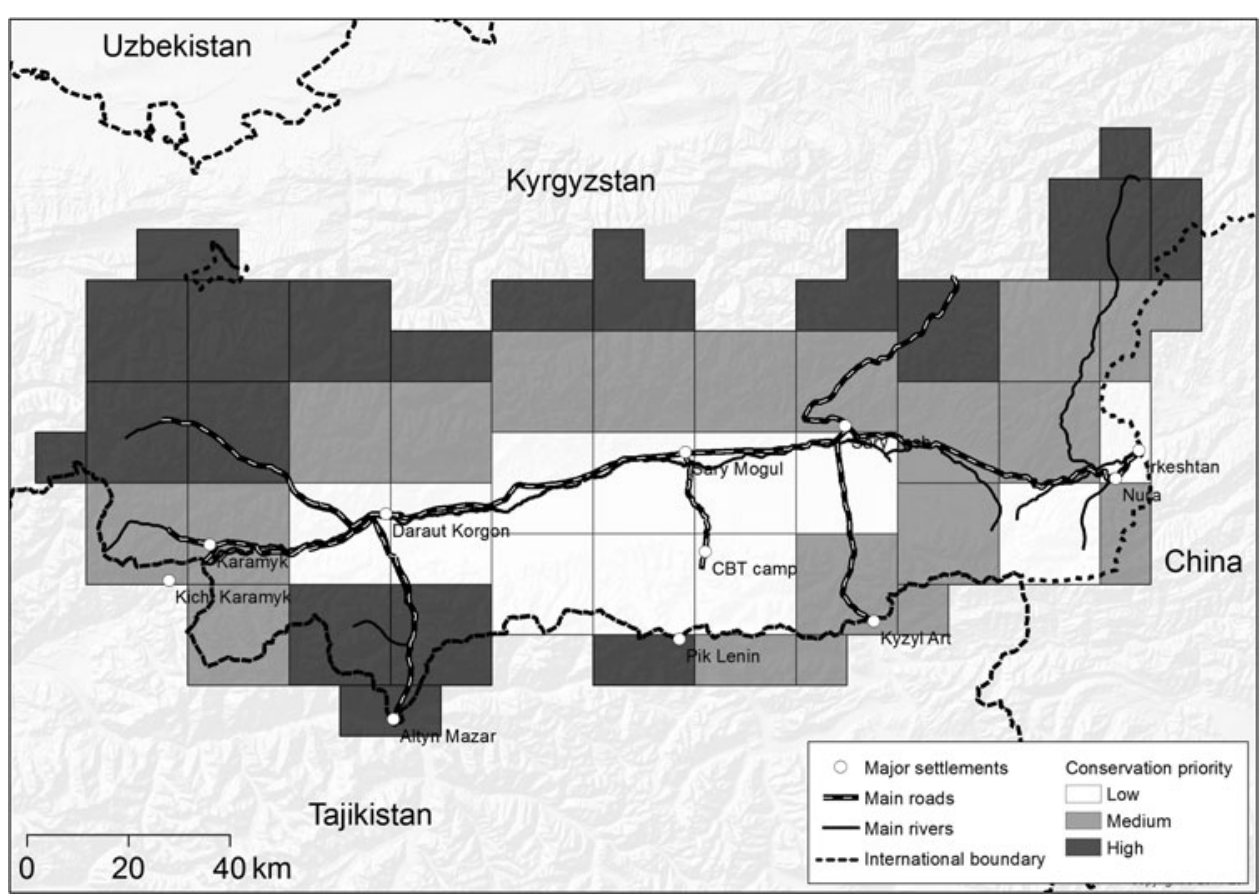

FIG. 3 Conservation priority areas in the Kyrgyz Alay Mountains (Fig. 1), based on the probabilities of current site use for snow leopard, brown bear, lynx and Marco Polo sheep. to use sites with lower mean slope and ruggedness $\left(\beta_{\text {ruggednessXslope }}=-6.09 \pm \mathrm{SE} 2.86\right)$, indicating rolling terrain. High probability $(>0.5)$ of local extinction was reported from $47 \%$ of sites. The probability of local extinction was greater around human settlements, with current distribution restricted to southern and eastern edges of Alay (Fig. 2h).

The species co-occurrence models did not yield any discernible pattern in the probability of occurrence of snow leopard and lynx that could indicate a mesopredator release. The coefficients of the parameters $\psi^{\mathrm{BA}}(\beta=-0.98 \pm$ SE 1.04) and $\psi^{\mathrm{Ba}}(\beta=-6.14 \pm$ SE 122,681.37) suggested the possibility of co-occurrence rather than opportunistic occupation by one species at the cost of another, but high variances indicated the inadequacy of the data for reliable interpretation.

\section{Discussion}

Dealing with false absences is crucial when reporting species distributions, local extinctions and colonizations. Nondetection may lead to underestimation of the area being used and overestimation of extinction probability. These errors are compounded if there are covariates influencing the parameters non-uniformly across the area of interest. We addressed the issue of imperfect detection by estimating probabilities of people detecting and reporting a species. Our results are derived from secondary data; we based our inferences on people's ability to recollect information about sightings of large mammals. Although we took adequate care when estimating probabilities of detection, site use, local extinction, and colonization, it is important that our results are interpreted with caution. The models are based on the assumption that species distribution did not change non-randomly during each primary sampling period (1990 and 2010). The models assumed that the probability of site use, detection probability, and variation between the primary sampling periods were a function of specific covariates that have been incorporated in the analysis. We did not consider the issue of false presence (Royle \& Link, 2006; Pillay et al., 2014), and although the possibility of confusing snow leopard and lynx was unlikely, this potential shortcoming could be addressed in primary surveys in the future. Our first sampling period coincided with the break-up of the Soviet Union, a watershed geopolitical event that influenced the status of biodiversity across the region. Our second sampling period was 2010; the Kyrgyz Republic has remained politically stable, and therefore we do not expect major changes in the status of biodiversity in the Alay Mountains to have occurred since then.

Our data suggest that most species, including the snow leopard, when present had low detection probabilities ( $\mathrm{P}_{\text {snow leopard: }}$ 0.08-0.13; $\mathrm{P}_{\text {Marco Polo }}$ 0.01-0.21; $\mathrm{P}_{\text {brown bear: }}$ $0.04-0.39 ; \mathrm{P}_{\text {lynx }}$ : 0.04-0.18). This makes the use of empirical methods to estimate detection probability particularly important. Analysis of the data on wolf and ibex was not deemed necessary, given their ubiquitous presence and detectability. The other four species appear to have declined in many parts of Alay since 1990, with the snow leopard, brown bear and Marco Polo sheep showing high probability of extinction $(\varepsilon>0.5)$ in $>35 \%$ of the sampled units. The snow leopard, which had a high probability of use in an estimated $73 \%$ of the sampling units in 1990 , appears to have lost more than a third of this range. However, our results suggest their continued persistence $(\psi>0.5)$ in an 
estimated $53 \%$ of the study area, which is higher than the distribution ( $21 \%$ of the area) reported by Watanabe et al. (2010), who also used an interview-based survey but did not take into consideration the possibility of imperfect detection of the species. The areas with high probability of extinction within Alay raise concerns about the remaining connectivity between the southern and northern global ranges of the snow leopard. We recommend that future studies should attempt to map population connectivity to the east, in China, and west of our study area along the Kyrgyz-Tajik border.

The lynx was the only species that, alongside local extinctions, also appeared to have colonized new areas. Although a weak correlation between the probability of colonization by lynx and the probability of extinction of the snow leopard indicated the possibility of mesopredator release (Crooks \& Soulé, 1999; Miller et al., 2001; Harihar et al., 2011; Ripple et al., 2013), our analysis of species co-occurrence did not support such a possibility. However, significant variance renders our interpretations tentative, and thus followup studies exploring such a pattern could be useful.

Most of the species we examined had a high probability of extinction in areas that were either adjacent to or within regions of high human use. These included areas of low altitude, ruggedness and slope, which are relatively more accessible to hunters. They also presumably attract keepers of livestock, and have undergone infrastructural development.

The large mammal populations of central Alay appear to have declined significantly since 1990, whereas in the northwest and on the northern and southern fringes of Alay the same species appear to have remained relatively stable (Fig. 3). The remnant population of Marco Polo sheep along the southern border may be persisting because of connectivity with larger populations in Tajikistan. Ours was a broad-scale study that identified areas with high probability of local extinction of four large mammals in Alay. Given the limitations of this preliminary work, intensive field surveys need to be undertaken to collect primary data, using sign surveys, camera trapping and collection of genetic material (e.g. scats) to understand the issues of connectivity and population abundance in the area.

\section{Acknowledgements}

We are grateful to Panthera, the Whitley Fund for Nature and the BBC Wildlife Fund for financial support that made this work possible. We thank James Nichols for reviewing our analytical methods and providing useful suggestions; F. Aitbaeva and G. Krippner for translation of the interviews, photographic documentation and sharing their knowledge; the 95 interviewees for their participation in this study; and the Editor and anonymous reviewers for their critical comments.

\section{References}

Anderson, D.R. \& Burnham, K.P. (2002) Avoiding pitfalls when using information-theoretic methods. Journal of Wildlife Management, 66, 912-918.

Asykulov, T. (2008) Social and Economic Issues in the Alay Region. Pamir-Alai Transboundary Conservation Area export report.

Bailey, L.L., Hines, J.E., Nichols, J.D. \& MacKenzie, D.I. (2007) Sampling design trade-offs in occupancy studies with imperfect detection: examples and software. Ecological Applications, 17, 281-290.

Bailey, L.L., Simons, T.R. \& Pollock, K.H. (2004) Estimating site occupancy and species detection probability parameters for terrestrial salamanders. Ecological Applications, 14, 692-702.

Brashares, J.S. (2003) Ecological, behavioral, and life-history correlates of mammal extinctions in West Africa. Conservation Biology, 17, 733-743.

Cabeza, M., Araújo, M.B., Wilson, R.J., Thomas, C.D., Cowley, M.J.R. \& Moilanen, A. (2004) Combining probabilities of occurrence with spatial reserve. Journal of Applied Ecology, 41, 252-262.

Cardillo, M., Mace, G.M., Jones, K.E., Bielby, J., Bininda-Emonds, O.R.P., Sechrest, W. et al. (2005) Multiple causes of high extinction risk in large mammal species. Science, 309, 1239-1241.

Cardillo, M., Purvis, A., Sechrest, W., Gittleman, J.L., Bielby, J. \& MACE, G.M. (2004) Human population density and extinction risk in the world's carnivores. PLoS Biology, 2(7), e197.

CGIAR-CSI (CGIAR Consortium for Spatial Information) (2004) Http://srtm.csi.cgiar.org/ [accessed 4 June 2015].

Crooks, K.R. \& Soulé, M.E. (1999) Mesopredator release and avifaunal extinctions in a fragmented system. Nature, 400, 563-566.

Farrington, J. (2005) A Report on Protected Areas, Biodiversity, and Conservation in the Kyrgyztan Tian Shan, with Brief Notes on the Kyrgyzstan Pamir-Alai and the Tian Shan Mountains of Kazakhstan, Uzbekistan and China. U.S. Fulbright Program, Environmental Studies Section, Bishkek, Kyrgyz Republic.

Global LAND Cover Facility (2010) Http://glcf.umd.edu/ [accessed 4 June 2015].

Harihar, A., Pandav, B. \& Goyal, S.P. (2011) Responses of leopard Panthera pardus to the recovery of a tiger Panthera tigris population. Journal of Applied Ecology, 48, 806-814.

Hines, J. (2006) PRESENCE: software to estimate patch occupancy and related parameters. Http://www.mbr-pwrc.usgs.gov/software/ presence.html [accessed4June2015].

Izumiyama, S., Anarbaev, M. \& Watanabe, T. (2009) Inhabitation of larger mammals in the Alai Valley of the Kyrgyz Republic. Geographical Studies, 84, 14-21.

Jackson, R. \& Hunter, D.O. (1996) Snow Leopard Survey and Conservation Handbook. 2nd edition. International Snow Leopard Trust, Seattle, USA, and U.S. Geological Survey, Colorado, USA.

Jackson, R.M., Roe, J.D., Wangchuk, R. \& Hunter, D.O. (2006) Estimating snow leopard population abundance using photography and capture-recapture techniques. Wildlife Society Bulletin, 34, $772-781$.

JanečKa, J.E., Munkhtsog, B., Jackson, R.M., Naranbaatar, G., Mallon, D.P. \& Murphy, W.J. (2011) Comparison of noninvasive genetic and camera-trapping techniques for surveying snow leopards. Journal of Mammalogy, 92, 771-783.

Jumabay-Uulu, K., Wegge, P., Mishra, C. \& Sharma, K. (2014) Large carnivores and low diversity of optimal prey: a comparison of the diets of snow leopards Panthera uncia and wolves Canis lupus in Sarychat-Ertash Reserve in Kyrgyzstan. Oryx, 48, 529-535. 
Karanth, K.U., Gopalaswamy, A.M., Kumar, N.S., Vaidyanathan, S., Nichols, J.D. \& MacKenzie, D.I. (2011) Monitoring carnivore populations at the landscape scale: occupancy modelling of tigers from sign surveys. Journal of Applied Ecology, 48, $1048-1056$.

Koshikarev, E. \& Vyrypaev, V. (2000) What has happened to the snow leopard since the break-up of the Soviet Union? Cat News, 32, 9-11.

Lunney, D., Crowther, M.S., Shannon, I. \& Bryant, J.V. (2009) Combining a map-based public survey with an estimation of site occupancy to determine the recent and changing distribution of the koala in New South Wales. Wildlife Research, 36, 262-273.

MacKenzie, D.I., Bailey, L.L. \& Nichols, J.D. (2004) Investigating species co-occurrence patterns when species are detected imperfectly. Journal of Animal Ecology, 73, 546-555.

MacKenzie, D.I. \& Nichols, J.D. (2004) Occupancy as a surrogate for abundance estimation. Animal Biodiversity and Conservation, $27,461-467$.

MacKenzie, D.I., Nichols, J.D., Hines, J.E., Knutson, M.G. \& Franklin, A.B. (2003) Estimating site occupancy, colonization, and local extinction when a species is detected imperfectly. Ecology, 84, 2200-2207.

MacKenzie, D.I., Nichols, J.D., Sutton, N., Kawanishi, K. \& BAILEY, L.L. (2005) Improving inferences in population studies of rare species that are detected imperfectly. Ecology, 86, 1101-1113.

MacKenzie, D.I. \& Royle, J.A. (2005) Designing occupancy studies : general advice and allocating survey effort. Journal of Applied Ecology, 42, 1105-1114.

Majıт, K. (2012) Physical Geography of the Kyrgyzstan. Bishkek, Kyrgyz Republic.

McCarthy, K.P., Fuller, T.K., Ming, M., McCarthy, T.M., Waits, L. \& Jumabaev, K. (2008) Assessing estimators of snow leopard abundance. The Journal of Wildlife Management, 72, 1826-1833.

McCarthy, T., Murray, K., Sharma, K. \& Johansson, O. (2010) Preliminary results of a long-term study of snow leopards in South Gobi, Mongolia. Cat News, 53, 15-19.

Miller, B., Dugelby, B., Foreman, D., Martinez, C., Noss, R., Phillips, M. et al. (2001) The importance of large carnivores to healthy ecosystems. Endangered Species Update, 18, 202-210.

Murray, M. (2008) Support to the Establishment of the Pamir-Alai Transboundary Conservancy Area Between Kyrgyzstan and Tajikistan (PATCA Project): Biodiversity Surveys of 2007. Project report.

National Statistical Committee (2010) Population and housing census 2009. In Book 3 (in tables). Provinces of Kyrgyzstan: Osh Province. National Statistical Committee of the Kyrgyz Republic, Bishkek, Kyrgyz Republic.

Pillay, R., Johnsingh, A.J.T., Raghunath, R. \& Madhusudan, M.D. (2011) Patterns of spatiotemporal change in large mammal distribution and abundance in the southern Western Ghats, India. Biological Conservation, 144, 1567-1576.

Pillay, R., Miller, D.A.W., Hines, J.E., Joshi, A.A. \& Madhusudan, M.D. (2014) Accounting for false positives improves estimates of occupancy from key informant interviews. Diversity and Distributions, 20, 223-235.

Ripple, W.J., Wirsing, A.J., Wilmers, C.C. \& Letnic, M. (2013) Widespread mesopredator effects after wolf extirpation. Biological Conservation, 160, 70-79.

Royle, J.A. \& Link, W.A. (2006) Generalized site occupancy models allowing for false positive and false negative errors. Ecology, 87, $835-841$.
Ruell, E.W., Riley, S.P.D., Douglas, M.R., Pollinger, J.P. \& CROOKs, K.R. (2009) Estimating bobcat population sizes and densities in a fragmented urban landscape using noninvasive capture-recapture sampling. Journal of Mammalogy, 90, 129-135.

SAGIMBAEV, S. (2007) PATCA expert reports: The mammals of the Alay Valley. NABU, Berlin, Germany.

Sappington, J.M., Longshore, K.M. \& Thompson, D.B. (2007) Quantifying landscape ruggedness for animal habitat analysis: a case study using bighorn sheep in the Mojave Desert. The Journal of Wildlife Management, 71, 1419-1426.

Sharma, K., Bayrakcismith, R., Tumursukh, L., Johansson, O., Sevger, P., McCarthy, T. \& Mishra, C. (2014) Vigorous dynamics underlie a stable population of the Endangered snow leopard Panthera uncia in Tost Mountains, South Gobi, Mongolia. PLoS ONE, 9(7), e101319.

Smith, J.B., Jenks, J.A. \& Klaver, R.W. (2007) Evaluating detection probabilities for American marten in the Black Hills, South Dakota. The Journal of Wildlife Management, 71, 2412-2416.

Snow Leopard Working Secretariat (2013) Global Snow Leopard and Ecosystem Protection Program. Snow Leopard Working Secretariat, Bishkek, Kyrgyz Republic. Http://www. snowleopardnetwork.org/sln/docs/Final_GSLEP_14_\%2011_\%2013. pdf [accessed 1 June 2015].

Symonds, M.R.E. \& Moussalli, A. (2011) A brief guide to model selection, multimodel inference and model averaging in behavioural ecology using Akaike's information criterion. Behavioral Ecology and Sociobiology, 65, 13-21.

van der Hoeven, C.A., De Boer, W.F. \& Prins, H.H.T. (2004) Pooling local expert opinions for estimating mammal densities in tropical rainforests. Journal for Nature Conservation, 12, 193-204.

Vorobeev, G. \& VAn Der Ven, J. (2003) Looking at Mammals in Kyrgyzia. Daru Ltd., Doorn, The Netherlands.

Watanabe, T., Izumiyama, S., Gaunavinaka, L. \& Anarbaev, M. (2010) Wolf depredation on livestock in the Pamir. Geographical Studies, 85, 26-36.

White, P.C.L., Jennings, N.V., Renwick, A.R. \& Barker, N.H.L. (2005) Questionnaires in ecology: a review of past use and recommendations for best practice. Journal of Applied Ecology, 42, 421-430.

Yanushevich, A.I., Aisin, B.M. \& Kydyraliev, A.K. (1972) Mammals of Kyrgyzia. Ilym Press, Frunze, Tajikistan. [In Russian]

Zeller, K.A., Nijhawan, S., Salom-Pérez, R., Potosme, S.H. \& Hines, J.E. (2011) Integrating occupancy modeling and interview data for corridor identification: a case study for jaguars in Nicaragua. Biological Conservation, 144, 892-901.

\section{Biographical sketches}

JULIA TAUBMANN is a wildlife biologist interested in wildlife research and conservation management, with a particular focus on felids, canids and birds. Koustubh Sharma is an ecologist; his main areas of interest are population and behavioural ecology, geographical information systems, and ecological modelling and training. KUBANYCHBEK Zhumabai UUlu is based in Bishkek and has worked for several years on snow leopards and other sympatric species in the Kyrgyz Republic. He has a keen interest in community-based conservation programmes. JAMES Hines specializes in writing code to analyse wildlife data. CHARUDUT T Mishra leads a programme aimed at securing the future of high-altitude mountain ecosystems by integrating indigenous communities with conservation efforts. 